\title{
Liste des thèses d'anthropologie soutenues en 2011
}

\section{Université de la Méditerranée Aix-Marseille-II}

Virginie FABRE : Réponse démographique des Néandertaliens face aux pressions environnementales du stade isotopique 3 : approche par modélisation écologique

Gaëlle GRANIER: Approche archéo-anthropologique des ensembles funéraires de l'Antiquité tardive. L'exemple des sites urbains de Vienne et Arles (III ${ }^{e}-I^{e}$ siècles)

Gianluigi MANGIAPANE : Analyse des données généalogiques d'une population du Limousin (France)

Julien SASTRE : Ontogenèse du crâne fretal : applications à la détermination de l'âge et du sexe

Delphine TARDIVO : Détermination de l'âge et du sexe et modélisation de la canine en anthropologie médicolégale

Majda CHERKAOUI : L'intégration des immigrées marocaines vieillissant seules en France et en Belgique. Quelle perception ont-elles de leur vie et de leur avenir au regard des politiques publiques mises en æuvre dans les deux pays d'accueil?

\section{Université Bordeaux-I}

Frédérique BLAIZOT: Les espaces funéraires de l'habitat groupé des Ruelles à Serris $d u V_{I I}^{e}$ au XIt siècle (Seine-et-Marne, Ille-de-France) : taphonomie du squelette, mode d'inhumation, organisation et dynamique

Pierre GUYOMARC'H : Reconstitution faciale par imagerie $3 D$ : variabilité morphométrique et mise en cuvre informatique

Célimène MUSSINI : Les restes humains moustériens des Pradelles (Marillac-le-Franc, Charente, France): étude morphométrique et réflexions sur un aspect comportemental des Néandertaliens

Aline THOMAS : Identités funéraires, variants biologiques et facteurs chronologiques : une nouvelle perception $d u$ contexte culturel et social du Cerny (Bassin parisien, 4700-4300 avant J.-C.)

\section{Université Toulouse-III}

Mélanie CAPREDON : Histoire biologique d'une population du Sud-Est malgache : les Antemoro

Michael COQUERELLE : Analyse tridimensionnelle $d u$ système dentomandibulaire des hommes modernes et des chimpanzés (Pan troglodytes) au cours de l'ontogenèse

Magdalena GAYÀ VIDAL: Genetic characteristics of the two main native groups in Bolivia: Aymaras and Quechuas

Marie LACAN : La néolithisation du bassin méditerranéen : apports de l'ADN ancien

Fanny MENDISCO : Apports de la paléogénétique à l'histoire du peuplement précolombien des Andes méridionales $\left(r^{\circ}-X v^{e}\right.$ siècles)

\section{Muséum national d'histoire naturelle}

Catherine LEMA: L'exploitation illégale du palmier protégé Euterpe edulis Mart. : dynamique d'un conflit environnemental dans le Parque Estadual Três Picos (État de Rio de Janeiro, Brésil)

Laurent PUYMERAIL: Caractérisation de l'endostructure et des propriétés biomécaniques de la diaphyse fémorale : la signature de la bipédie et la reconstruction des paléorépertoires posturaux et locomoteurs des homininés

Rihlat SAID MOHAMED : Adaptation à la malnutrition dans les sociétés en transition nutritionnelle. Étude des déterminants biologiques, sociaux et culturels du développement du surpoids chez les enfants en retard de croissance à Yaoundé (Cameroun)

Clément ZANOLLI : L'organisation endostructurale des restes dentaires humains du Pléistocène inférieur final - moyen initial d'Indonésie et d'Afrique, avec une attention particulière à Homo erectus s.s. Caractérisation comparative à haute résolution et problématiques taxinomiques 